\title{
Some Operations on Mixed Monotone Operator in Banach Spaces
}

\author{
Badrulfalah $^{1^{*}}$, I Irianingsih ${ }^{2}$, Khafsah $\mathbf{J}^{3}$ \\ ${ }^{1 *}$ Mathematics Department, FMIPA, Universitas Padjajaran \\ ${ }^{2}$ Mathematics Department, FMIPA, Universitas Padjajaran \\ ${ }^{3)}$ Mathematics Department, FMIPA, Universitas Padjajaran \\ *Email : badrulfalah@gmail.com
}

\begin{abstract}
This paper discusses some operations on mixed monotone operator in Banach space, especially addition an multiplication operations. We will prove the sum and product of two mixed monotone operators. The proof using some relevant definitions. The result is the sum o of them is a mixed monotone operator and the product is too if both satisfy some conditions.
\end{abstract}

Keywords : non-decreasing and non-increasing, Mixed monotone operator, Banach space, partial ordering.

\section{Introduction}

There are many classes of real functions. One of them is a monotone function. On the other side, we have known a term called mixed monotone operator, that is a function defined on the Cartesian Product of two subsets of Real Banach Space[1-9, 13]. It has been well-known that under operation of addition, the sum of two monotone functions is monotone function too, but not for case of multiplication operation[10]. Inspired by both of result above, we interested on knowing how about addition and multiplication of two mixed monotone operator defined in real Banach space. Hence, we will prove it here. The objective of this research is to prove the sum and product of two mixed monotone operators and to find some conditions in order to the product is mixed monotone operator.

\section{Research Method}

To prove the sum and product of two mixed monotone operators done in three steps. Firstly by defining some partial ordering in subset of Banach space. Then to find some conditions of mixed monotone operators. The last showing that two relevant operations satisfy some definition.

\section{Result and Discussion}

\section{Prelimineries}

In this section we will give some definitions and theories of monotone function.

Definition 1.1: Let $S$ be a nonvoid set and $R$ is relation on set $S$. Relation $R$ is called partial ordering on $S$ if $R$ satisfy the following property:

(i) $R$ is reflexive

(ii) $R$ is antysimmetric

(iii) $R$ is transitive [3] 
Definition 1.2: Let $E$ always be a Real Banach space and $P$ a subset of $E$. $P$ is called a cone if

(i) $P$ is closed, non-empty and $P \neq\{0\}$

(ii) $a x+b y \in P$ for all $x, y \in P$ and nonnegative real numbers $a, b$

(iii) $P \cap(-P)=\{0\}[11]$

Definition 1.3 : Let $X$ be a nonvoid set and let $E$ be a Real Banach space equipped with the partial oredering $\leq$ with respect to the cone $P \subseteq E$. Suppose that the mapping $d: X \times X \rightarrow E$ satisfies the following conditions:

$\left(d_{1}\right) 0 \leq d(x, y)$ for all $x, y \in X$ and $d(x, y)=0$ if and only if $x=y$;

$\left(d_{2}\right) d(x, y)=d(y, x)$ for all $x, y \in X$;

$\left(d_{3}\right) d(x, y) \leq d(x, z)+d(z, y)$ for all $x, y, z \in X .[11]$

Then $\boldsymbol{d}$ is called a cone metric on $X$, and $(X, d)$ is called a cone metric space.

Definition $1.4:$ Let $S \subset \mathbb{R}$. A function $f: S \rightarrow \mathbb{R}$ is said to be nondecreasing on $S$ if whenever $x, y \in S$ and $x<y$ then $f(x) \leq f(y)[10]$.

Definition 1.5: Let $S \subset \mathbb{R}$. A function $f: S \rightarrow \mathbb{R}$ is said to be nondecreasing on $S$ if whenever $x, y \in S$ and $x<y$ then $f(y) \leq f(x)[10]$.

The following Lemma 1.6 state the result of the sum of two monotone functions.

Lemma 1.6: Let $S \subset \mathbb{R}$ and two functions $f, g: S \rightarrow \mathbb{R}$. If $f$ and $g$ are monotone functions then $f+g$ is a monotone function.[10]

The following Example 1.7 illustrate the product of two monotone functions in not monotonous. Example 1.7: Let $f(x)=x$ and $f(x)=x g(x)=x-1$ defined on $[0,1]$, then $f$ and $g$ are nondecreasing functions on $[0,1]$ but their product $f g$ is not nondecreasing on $[0,1]$.[1]

Let $E$ is a Real Banach space, which is partially ordered by a cone $P$, i.e., $x \leq y$ iff $y-x \in P$. Definition 1.8: Let $D \subset E$. An operator $A: D \times D \rightarrow E$ is said to be mixed monotone if $A(x, y)$ is nondecreasing in $x$ and nonincreasing in $y$.[13]

\section{Main Result}

In this part we assume $\mathrm{E}$ is real Banach space which is partially ordered by a cone $\mathrm{P}$, put $\tilde{P}=$ $\{(x, y) \in E \times E \mid x \geq 0, y \leq 0\}$. It is clear that $\tilde{P}$ is a cone in $E \times E$.

We need the following Lemma 2.1 to prove two next theorems as main result.

Lemma 2.1 : Define relation $\preccurlyeq$ on $E \times E$ by $\left(x_{1}, y_{1}\right) \preccurlyeq\left(x_{2}, y_{2}\right)$ if and only if $x_{1} \leq x_{2}$ and $y_{1} \geq y_{2}$. Then $\leqslant$ is partial ordering in $E \times E$.

\section{Proof:}

To prove that $\preccurlyeq$ is partial ordering in $E \times E$ firstly we show that the relation $\leq$ on $E \times E$ is reflexsive. 
Let $(x, y)$ be arbitrary in $E \times E$ then we get $x \leq x$ and $y \geq y$. Following defining of relation $\preccurlyeq$, it means $(x, y) \preccurlyeq(x, y)$. In according to Definition 1.1.( $i)$, then the relation $\preccurlyeq$ is reflexsive.

For the condition (ii) dan (iii), we leave it.

Let Let $D \subset E$ and operator $A, B: D \times D \rightarrow E$ be mixed monotone operators.

The sum of two mixed monoton operaton in the following Theorem 2.2.

Theorem 2.2: Let Let $D \subset E$ and operator $A, B: D \times D \rightarrow E$. If $A$ and $B$ are mixed monotone operators then $A+B$ is a mixed monotone operator.

Proof: To prove that $A+B$ is a mixed monotone operator, firstly we show that $A+B$ is nondecreasing in $x$.

Suppose $\left(x_{1}, y\right)$ and $\left(x_{2}, y\right) \in D \times D$ with $\left(x_{1}, y\right) \preccurlyeq\left(x_{2}, y\right)$. Based on Lemma 2.1 then $x_{1}<x_{2}$. Since $A$ and $B$ are mixed monotone operators, following Definition 1.8 then $A$ and $B$ are nondecreasing in $x$. Based on Definition 1.4 we get $A\left(x_{2}, y\right)-A\left(x_{1}, y\right) \geq 0$ and $B\left(x_{2}, y\right)-$ $B\left(x_{1}, y\right) \geq 0$. Therefore

$(A+B)\left(x_{2}, y\right)-(A+B)\left(x_{1}, y\right) \geq 0$.

So $(A+B)\left(x_{2}, y\right) \geq(A+B)\left(x_{1}, y\right)$.

In according to Definition 1.4, we conclude $A+B$ is nondecreasing in $x$.

The last, we show that $A+B$ is nondecreasing in $y$.

Suppose $\left(x, y_{1}\right)$ and $\left(x, y_{2}\right) \in D \times D$ with $\left(x, y_{1}\right) \preccurlyeq\left(x, y_{2}\right)$. Based on Lemma 2.1 then $y_{1}>y_{2}$. Since $A$ and $B$ are mixed monotone operators, following Definition 1.8 then $A$ and $B$ are nonincreasing in $y$. Based on Definition 1.4 we get $A\left(x, y_{2}\right)-A\left(x, y_{1}\right) \geq 0$ and $B\left(x, y_{2}\right)-$ $B\left(x, y_{1}\right) \geq 0$. Therefore

$(A+B)\left(x, y_{2}\right)-(A+B)\left(x, y_{1}\right) \geq 0$. So $(A+B)\left(x, y_{2}\right) \geq(A+B)\left(x, y_{1}\right)$. In according to Definition 1.4, we conclude $A+B$ is nonincreasing in $y$.

Hence, in according to Definition 1.8, we conclude that $A+B$ is a mixed monotone operator.

The result of the product of two mixed monotone operators given in the following Theorem 2.3.

Theorem 2.3: Let Let $D \subset E$ and operator $A, B: D \times D \rightarrow E$. If $A$ and $B$ are positive mixed monotone operators then $A+B$ is a mixed monotone operator.

Proof: To prove that $A B$ is a mixed monotone operator, firstly we show that $A B$ is nondecreasing in $x$.

Suppose $\left(x_{1}, y\right)$ and $\left(x_{2}, y\right) \in D \times D$ with $\left(x_{1}, y\right) \preccurlyeq\left(x_{2}, y\right)$. Based on Lemma 2.1 then $x_{1}<x_{2}$. Since $A$ and $B$ are mixed monotone operators, following Definition 1.8 then $A$ and $B$ are nondecreasing in $x$. Based on Definition 1.4 we get $A\left(x_{2}, y\right)-A\left(x_{1}, y\right) \geq 0$ and $B\left(x_{2}, y\right)-$ $B\left(x_{1}, y\right) \geq 0$. Therefore

$A B\left(x_{2}, y\right)-A B\left(x_{1}, y\right)=A\left(x_{2}, y\right) B\left(x_{2}, y\right)-A\left(x_{2}, y\right) B\left(x_{1}, y\right)+A\left(x_{2}, y\right) B\left(x_{1}, y\right)-$ $A\left(x_{1}, y\right) B\left(x_{1}, y\right)$.

$=A\left(x_{2}, y\right)\left[B\left(x_{2}, y\right)-B\left(x_{1}, y\right)\right]-\left[A\left(x_{2}, y\right)-A\left(x_{1}, y\right)\right] B\left(x_{1}, y\right)$

Because $A$ and $B$ are positive operators then $A B\left(x_{2}, y\right)-A B\left(x_{1}, y\right) \geq 0$.

So, $A B\left(x_{2}, y\right) \geq A B\left(x_{1}, y\right)$.

In according to Definition 1.4 , we conclude $A B$ is nondecreasing in $x$.

The last, we show that $A B$ is nonincreasing in $y$. 
Suppose $\left(x, y_{1}\right)$ and $\left(x, y_{2}\right) \in D \times D$ with $\left(x, y_{1}\right) \preccurlyeq\left(x, y_{2}\right)$. Based on Lemma 2.1 then $y_{1}>y_{2}$. Since $A$ and $B$ are mixed monotone operators, following Definition 1.8 then $A$ and $B$ are nonincreasing in $y$. Based on Definition 1.4 we get $A\left(x, y_{2}\right)-A\left(x, y_{1}\right) \geq 0$ and $B\left(x, y_{2}\right)-$ $B\left(x, y_{1}\right) \geq 0$. Therefore

$A B\left(x, y_{2}\right)-A B\left(x, y_{1}\right)=A\left(x, y_{2}\right) B\left(x, y_{2}\right)-A\left(x, y_{2}\right) B\left(x, y_{1}\right)+A\left(x, y_{2}\right) B\left(x, y_{1}\right)-$ $A\left(x, y_{1}\right) B\left(x, y_{1}\right)$.

$=A\left(x, y_{2}\right)\left[B\left(x, y_{2}\right)-B\left(x, y_{1}\right)\right]-\left[A\left(x, y_{2}\right)-A\left(x, y_{1}\right)\right] B\left(x, y_{1}\right)$

Because $A$ and $B$ are positive operators then $A B\left(x, y_{2}\right)-A B\left(x, y_{1}\right) \geq 0$.

So $A B\left(x, y_{2}\right) \geq A B\left(x, y_{1}\right)$.

In according to Definition 1.4 , we conclude $A B$ is nondecreasing in $y$.

Hence, in according to Definition 1.8, we conclude that $A B$ is a mixed monotone operator.

\section{Conclusion}

In this paper we have the conlusion that the sum of two mixed monotone operator is mixed monotone operator, while the product is mixed monotone operator if both are positive operator.

\section{References}

[1] Wei L, Agarwal RP. 2018. Simple form of a projection set in hybrid iterative schemes for non-linear mappings, application of inequalities and computational experiments. Journal of inequalities and applications 2018:179

[2] Wei L, Agarwal RP. 2018. New construction and proof techniques of projection algorithm for countable maximal monotone mappings and weakly relatively nonexpansive mappings in a Banach space. Journal of inequalities and applications 2018:64

[3] Lochowski RM. 2018. A new inequality for the Riemann-Stieltjes integrals driven by irregular signals in Banach spaces. Journal of inequalities and applications 2018:20

[4] Farid M, Irfan SS, Khan MF, Khan SA. 2017. Strong convergence of gradient projection method for generalized equilibrium problem in a Banach space. Journal of inequalities and applications 2017:297

[5] Wei L, Duan L, Agarwal RP, Chen R, Zheng Y. 2017. Modified forward-backward splitting midpoint method with superposition perturbations for the sum of two kinds of infinite accretive mappings and its applications. Journal of inequalities and applications 2017:227

[6] Sarfaraz M, Ahmad MK, Kilicman A. 2017. Approximation solution for system of generalized ordered variational inclusions with plus sign in circle operator in ordered Banach space. Journal of inequalities and applications 2017:81

[7] Diop C, Sow TM, Djitte N, Chidume CE. 2015. Constructive techniques for zeros of monotone mappings in certain Banach spaces. SpringerPlus 4:383

[8] Li B, Yang D, Yuan W. 2014. Anisotropic hardy spaces of Musielak-Orlicz type with applications to boundedness of sublinear operators. TheScientificWorldJournal 2014:306214

[9] Bartle, Robert. 1992. Introduction to Real Analysis(2 ${ }^{\text {nd }}$ Edition). John Wiley \& Sons. Inc., New York. 
[10] Huang, L.G; Zhang, X. 2007. Cone Metric Spaces and Fixed Point Theorems of Contractive Mapping. Journal of Mathematical Analysis And Applications 332, No. 2, pp.1468-1476.

[11] Rosen, K. H. 1994. Discrete Mathematics and Its Applications(3nd Edition). McGrawHill, Inc., New York.

[12] Young-Zhou Chen. 1991. Existence of Coupled Fixed Points. Journal of Mathematical Analysis and applications, 154, pp.142-150. 